\title{
Association of red meat consumption with the incidence of cardiovascular disease
}

\author{
Steven Chrysant ${ }^{1}$ and George S. Chrysant ${ }^{2}$ \\ ${ }^{1}$ University of Oklahoma Health Sciences Center \\ ${ }^{2}$ INTEGRIS Baptist 7 Medical Center
}

June 11, 2020

\begin{abstract}
Background: Red meat, processed and unprocessed, has been associated with increased incidence of cardiovascular disease (CVD), stroke, heart failure (HF), and type 2 diabetes mellitus (T2DM). Despite these adverse effects, it is still, highly consumed by the people of developed counties and increasingly consumed by the people of developing countries. Aims: The aim of the paper was to review the current evidence on the effects of processed and unprocessed red meat consumption on the incidence of CVD, stroke, HF, and T2DM. Materials and Methods: A Medline search of the English language literature was conducted between 2010 and April 2020 using the terms, red meat, white meat, processed meat, unprocessed meat, cardiovascular disease, heart failure, diabetes mellitus and 22 pertinent papers were retrieved. Results: The analysis of results from these papers reveled that high red meat, especially processed meat consumption, is significantly associated with an increased risk of CVD, stroke, HF, and T2DM regardless of age, sex, or ethnicity. Discussion: The results of the studies retrieved, revealed that there is a controversy regarding the association of red meat consumption with an increased incidence of CVD. This controversy is due to the conflicting findings of the studies reviewed, with several studies showing no association, whereas others sowing an association, especially in subjects at high cardiovascular risk. Conclusion: There is regarding the association of red meat consumption with the incidence of CVD due to conflicting results of the various studies. Moderate red meat consumption showed no association with CVD in healthy subjects, but an association in high CVD risk subjects. Therefore, subjects at high cardiovascular risk should refrain from high red meat consumption and increase the intake healthier foods high in fiber content.
\end{abstract}

\section{Introduction}

Red meat, processed and unprocessed, is a favorable food staple of the people living in the Western countries including the US, Europe, Australia, and New/Zealand. In the US, it accounts for about $40 \%$ of the total daily red meat production [1]. Although there is a recent trend for decreased red meat consumption in the US, the trend is rising in the developing countries as their income is improving [2,3]. From a physiological point of view, red meat consumption has several nutritional benefits due to its content in protein, iron, zinc, folate, and vitamins $\mathrm{A}$ and $\mathrm{B}$, but also has certain drawbacks due its high content in total cholesterol (TC), low density lipoprotein cholesterol (LDL-C), and saturated fatty acids [4], The latter ones have been responsible for the increased incidence of atherosclerotic heart disease (ASHD), coronary heart disease (CHD), cardiovascular disease (CVD), type 2 diabetes mellitus (T2DM), strokes, heart failure (HF), and all-cause mortality [5-10]. In contrast, consumption of white meat, like poultry and fish, and perhaps, pork are negatively associated with the incidence risk of CVD and all-cause mortality [11,12]. In addition, several studies have confirmed that lowering the serum LDL-C with treatment will decrease the incidence ASCVD as well as the incidence of primary and secondary CVD [13-17]. A recent analysis of alternative food preferences by the American people showed that about $2 \%$ of them consumed a vegetarian type diet for health reasons and these people were mostly educated with greater than high school education, and were mostly female, non smokers, and physically active [18]. In addition, there has been recently, an increased trend for a shift for plant-based 
meat and for vegetarian and vegan diets, even among athletes $[19,20]$. In order to get a better perspective on the current consumption of unprocessed and processed red meat and its association with CVD, CHD, stroke, HF, and all-cause mortality, a focused Medline search of the English language literature was conducted between 2010 and April 2020, using the terms, processed, red meat, unprocessed meat, cardiovascular disease, coronary heart disease, stroke, heart failure, all-cause mortality and 22 pertinent papers were retrieved. These papers together with collateral literature will be discussed in this review.

\section{Studies showing a positive association of high consumption of processed and unprocessed red meat with the incidence of cardiovascular disease}

Several studies have demonstrated a positive association with high consumption of unprocessed and processed red meat and the incidence of CVD and cardiovascular (CV) mortality. These studies are listed in table 1 and they will be, briefly, discussed here. The study by Zheng et al [6], is a prospective cohort study of 81,469 US men and women ages 59-61 years on the effects of high consumption of unprocessed and processed red meat and incidence of CV mortality. After a mean follow-up of 14.7 years, the highest consumption ( $>05$ servings/day) of unprocessed red meat was associated with a $10 \%$ higher incidence of CV mortality, HR 1.10 $(95 \%$ CI 1.04- 1.17, p < 0.001)), whereas the highest consumption of processed meat was associated with a $13 \%$ higher incidence of CV mortality, HR 1.13 (95\% CI 1.04-1.23, p < 0.001) compared to those with the lowest meat consumption. In another prospective cohort study, Pan et al [21], analyzed the effects of red meat consumption on all-cause mortality in 121,342 men and women ages 52-54 free of CVD at baseline. After 22 years of follow-up and multivariate adjustments, one serving of unprocessed or processed red meat was associated with $13 \%$ and $20 \%$ increase in all-cause mortality, HR 1.13 (95\% CI 1.07-1.20), and 1.20 (95\% CI 1.15-1.24), respectively. The corresponding percentages for CV mortality were $18 \%$ and $21 \%$, HR 1.18 (95\% CI 1.13-1.23) and 1.21 (95\% CI 1.13-1.31) for unprocessed and processed red meat, respectively. Also, in a case-controlled study, Wang et al [22], examined the relationship between unprocessed, processed, and total red meat consumption with the incidence of CAD and fatal and nonfatal myocardial infarction (MI) in 2,131 Costa Rican patients mean age 58.1 years. After 10 years of follow-up, the odds ratio (OR) for the onset of acute MI were higher in the $5^{\text {th }}$ quintile $(110.8 \mathrm{~g} /$ day $)$ than the $1^{\text {st }}$ quintile, OR $1,29(95 \%$ CI 1.01-1.65) for processed meat and OR 1.31 (95\% CI 1.04-1.65) for total red meat consumption, respectively. Regarding the influence of sex on the incidence of MI, women had higher incidence than men, OR 1.47 (95\% CI 0.80-2.69). In another prospective cohort study, Key et al [23], examined the effects of meat on the incidence of ischemic heart disease (IHD) in 409,885 subjects ages 51-61 years, from the Pan-European Epic Cohort study. After 12.6 years of follow-up, the incidence of IHD between the top $5^{\text {th }}$ and bottom $5^{\text {th }}$ of unprocessed or processed red meat consumption was increased by $10 \%$ and $13 \%$, HR 1.10 (95\% CI 0.991.21) and HR 1.13 (95\% CI 1.02-1.26) for unprocessed and processed red meat, respectively. In contrast, the consumption of fish, poultry, milk, or eggs were not associated with an increased incidence of IHD. Similarly, a prospective cohort study by Alshahrami et al [24], examined the effects of unprocessed or processed red meat on the incidence of CV mortality and all-cause mortality in 96,000 subjects from the Seventh -day Adventist faith 53-57 years. After a mean follow-up of 11.8 years, unprocessed red meat was associated with increased incidence of CV mortality and all-cause mortality, HR 1.26 (95\% CI 1.05-1.50) and HR 1.18 (95\% CI 1.07-1.31), respectively. Processed red meat alone was not associated with an increased incidence of CV mortality, but the combination of unprocessed and processed red meat was, HR 1.23 (95\% CI 1.11-1.36). Also, a recent study by Zhong et al [25], analyzed the of data from 6 US prospective cohort studies of 29,682 subjects mean age 53.7 years, the effects of red meat, poultry, or fish consumption on the incidence of the composite endpoint of (CHD, stroke, HF, CVD, all-cause death). After 19 years of follow-up, there was a positive association of red meat (processed and unprocessed), and poultry, but not fish on the incidence of CVD and all-cause mortality. The adjusted HR (aHR) for CVD was 1.07 (95\% CI1.04-1.11) for processed red meat, aHR 1.03 (95\% CI 1.01-1.06) for unprocessed red meat, and aHR 1.04 (95\% CI 1.01-1.06) for poultry. Also processed and unprocessed red meat had a positive association with all-cause mortality, aHR 1.03 (95\% CI 1.02-1.05), aHR 1.03 (95\% CI 1.01-1.05), respectively. In contrast, poultry or fish consumption were not associated with an increase in all-cause mortality.

Similar effects were demonstrated by several reviews and meta-analyses. The review and meta-analysis by 
Micha et al [26], consists of 20 studies (17 prospective and 3 case control), involving 1,218,380 subjects on the effects of high meat consumption ( [?] $100 \mathrm{~g} /$ day) on the incidence of CHD. In this review, unprocessed red meat consumption was not associated with an increased incidence of CHD, HR 1.00 (95\% CI 0.81-1.23, $\mathrm{p}=0.36$ ) whereas, processed meat consumption was associated with a $42 \%$ higher risk of CHD, HR 1.42 (95\% CI 1.07-1.89, $\mathrm{p}=0.04)$. Associations were intermediate for total meat intake. In a review and metaanalysis of prospective studies involving 1,330,352 subjects ages 20-86 years, Larsson et al [27], analyzed the effects of unprocessed and processed red meat consumption on all-cause mortality. After a follow-up of 9-28 years and multivariate adjustment analyses, the highest vs the lowest consumption of unprocessed or processed red was associated with 10\%, HR 1.10 (95\% CI 0.98-1.22) and 29\%, HR 1.29 (95\% CI 1.24-1.35) higher incidence of ACM, respectively. The study by Bellavia et al [28], is a prospective study of 109,201 Swedish men and women ages 45-79 years. After a mean follow-up of 15 years, those subjects consuming $>$ $100 \mathrm{~g}$ of unprocessed meat/day had a shorter survival than those never consuming red meat. The HRs for total red meat consumption were HR 1.16 (95\% CI 1.06-1.127) for $150 \mathrm{~g} /$ day, HR 1.26 (95\% CI 1.14-1.40) for $200 \mathrm{~g} /$ day, HR 1.37 (95\% CI 1.21-1.36) for $250 \mathrm{~g} /$ day, and HR 1.49 (95\% CI 1.27- 1.75) for $300 \mathrm{~g} /$ day, respectively. This study demonstrated a dose-response association of total red meat consumption with short term survival. The review and meta-analysis of 13 prospective cohort studies involving 1,674,272 subjects ages 18-92 years, by Abete et al [29], concerns the association of consumption of unprocessed and processed red, white and total meat with mortality from CVD, IHD, and all-cause mortality. The analysis of results from these studies, showed that high consumption of unprocessed or processed red meat was associated with an increased incidence of CVD mortality, 16\% relative risk (RR), 1.16 (95\% CI 1.03-1.32, p < 001) for unprocessed red meat, and 18\%, RR 1.18 (95\% CI 1.05-1.32, p < 0.002) for processed meat, respectively. Regarding the IHD mortality, processed but not unprocessed red meat consumption, was associated with a statistically increased IHD mortality, RR $1.52(95 \%$ CI 0.50-4,66, p = 0.004) for processed and RR $1.02(95 \%$ CI 0.72-1.46) for unprocessed red meat, respectively. Also, total red meat, but not white meat consumption was associated with increased IHD mortality, RR 1.52 (95\% CI 0.68-3.40) for total and RR 1.00 (95\% CI 0.82-1.21) for white meat, respectively. Regarding the consumption of processed meat, this was associated with increased all-cause mortality, RR 1.22 (95\% CI 1.16-1.29), but not the total red and white meat consumption. Also, processed red meat was positively associated with increased all-cause mortality in both men and women.

\section{Studies Showing a Positive Association of Red Meat Consumption with the Incidence of Stroke}

Red meat consumption, besides increasing the incidence of CVD, is also associated with an increased incidence of stroke. Several reviews demonstrating this association are listed in table 2 and they will be briefly, discussed here. In a review of 5 cohort studies involving 239,251 subjects, Chen et al [30] analyzed the association of red meat consumption with the incidence of stroke. After a mean follow-up of 10 to 26 years, the pooled analysis of results showed an increased risk for total stroke in the subjects with a higher consumption of total red meat vs with a lower consumption of total red meat (unprocessed + processed), summary RR (sRR) 1.15 (95\% CI 1.05-1.25). In a dose-response analysis, the incidence of ischemic stroke increased by $10 \%$ for each $100 \mathrm{~g} /$ day increase in the consumption of total red meat and by $13 \%$ increase in consumption of unprocessed red meat. In addition, for each $50 \mathrm{~g}$ increase in the consumption of processed meat the incidence of stroke increased by $11 \%$. In another review and meta-analysis of 7 prospective studies involving 2,o79,236 subjects ages 30-33 years, Yang et al [31], analyzed the effects of unprocessed red on the incidence of stroke. After a mean follow-up of 10 to 26 years (by different studies), the risk of ischemic stroke between the highest and lowest consumption of unprocessed red meat was 15\%, RR $1.15 * 95 \%$ CI 1.03-1.29) and with consumption of total red meat, RR 1.22 (95\% CI 1.01-1.28). In addition, the consumption of unprocessed red meat was associated with a $13 \%$ higher incidence of cerebral infarction, RR 1.13(95\% CI 1.01-1.28). In contrast, the consumption of total meat was associated with $22 \%$ higher incidence of ischemic stroke, RR 1.22 (95\% CI 1.01-1.46). In a dose-response analysis of results, there was not a risk of stroke if the consumption of total red meat was lower than $50 \mathrm{~g} /$ day, but higher if the consumption was greater than $50 \mathrm{~g} /$ day. The review and meta-analysis by Kim et al [32], included 15 studies involving 254,742 subjects ages 30-83 on the effects of red meat on the incidence of stroke. After a mean follow-up of 5.5 to 26 years by the different studies, 
the pooled RR (pRR) for the incidence of total stroke with consumption of unprocessed, processed, an total red meat was, 1.11 (95\% CI 1.03- 1.20), 1.17 (95\% CI 1.08-1.25), and 1.18 (95\% CI 1.09-1.28), respectively. In contrast, the pRR for white meat consumption was 0.87 (95\% CI 0.78- 0.97). This study demonstrated that white meat consumption was not associated with an increased incidence of stroke compared to red meat consumption.

\section{Studies Showing a Positive Association of Red Meat Consumption with the Incidence of Heart Failure}

Red meat consumption besides its association with CVD and stroke, has also been associated with an increased incidence of HF. The studies demonstrating this association are listed in table 3 and they will be, briefly, discussed here. The study by Ashaye et al [33], is a prospective cohort study of 21,120 healthy men from the Physician's Health Study, mean age 54.6 years. Red meat consumption was assessed by abbreviated questionnaires, and the onset of HF was assessed through follow-up questionnaires. After a mean follow-up of 19.9 years, meat consumption (beef, pork, or lamb), the HRs for new incidence of HF between the lowest consumption (quintile 1 as standard) and quintiles 2,3,4 and 5 (highest consumption), were 1.01 (95\% CI 0.85-1.22), 1.12 (95\% CI 0.93-1.34), 1.22 (95\% CI 1.01-1.47), and 1.37 (95\% CI 1.14- 1.64), respectively ( $\mathrm{p}<$ 0.0001). However, after adjustment for other risk factors, the incidence of HF showed a decreasing trend, HR 1.22 (95\% CI 1.03-1.48) for quintile 5 compared to quintiles 2-4 (p < 007). In this study, the association of HF was assessed with and without the incidence of MI. The study by Kaluza et al [34], is a prospective cohort study on the association of unprocessed or processed red meat consumption on the incidence of HF in 37,035 Swedish men mean age 45-79 years, free of HF, or ischemic stroke at baseline. After a mean follow-up of 11.8 years, processed meat was significantly associated with the risk of HF. Men who consumed [?] 75 $\mathrm{g} /$ day of processed meat had a significantly higher risk of $\mathrm{HF}$ compared to those consuming $<25 \mathrm{~g} /$ day, $\mathrm{HR}$ 1.28 (95\% CI 1.10-1.48, $\mathrm{p}<0.01$ for trend) and also, a higher risk of mortality from HF, HR 2.43 (95\% CI $1.52-3.88, \mathrm{p}<0.001$ for trend). In contrast, the consumption of unprocessed red meat was not significantly associated with an increased risk of HF or death. In a similar prospective cohort study, Kaluza et al [35] investigated the effects of red consumption on the incidence of HF in 34,057 Swedish women aged 48-83 years. After a mean follow-up of 13.2 years, the consumption of processed red meat [?] $50 \mathrm{~g} /$ day compared to $<25$ $\mathrm{g} /$ day, was associated with a greater risk of HF, short and log-term, HR 1.23 (95\% CI 1.09-1.39, P $<0.003$ for trend) and HR 1.78 (95\% CI 1.00-3.16), respectively. In contrast, consumption of unprocessed red meat was not associated with an increased risk of HF. The study by Cui et al [36], is a review and meta-analysis of 6 studies involving 134,863 subjects ages 35-83 years, on the effects of red meat consumption on the incidence of HF. After a mean follow-up of 8.2-21.5 years, a significant association with the incidence of HF was found between the highest vs the lowest consumption of processed meat, RR 1.33 (95\% CI 1.15-1.54) for the European subjects, but not for the American subjects. In contrast, unprocessed red meat consumption was not significantly associated with an increased risk of HF, RR 1.04 (95\% CI 0.96-1.12).

\section{Studies Showing an Association of Red Meat Consumption and T2DM}

Several studies have also, shown an association between unprocessed or processed red meat consumption and incidence of T2DM. These studies are summarized in table 4 and they will be briefly, discussed here. The study by Liu et al [37], is prospective cohort study of 59,033 women ages 30-55 years, free of CVD and T2DM at baseline. After a mean follow-up of 17.3 years, and multivariate adjustments for red meat cooking methods, total red meat intake, processed or unprocessed [?] 2 times/week vs $<1$ time/month, were associated with an increased risk of T2DM $(\mathrm{p}<0.05)$ for all. However, the higher frequency of broiling it or barbequing it had a greater impact on the risk of T2DM $(\mathrm{p}<0.001)$ compared to stewing or boiling it. This study demonstrated that high temperature or open-flame cooking of red meat, may further increase the risk for T2DM. The study by Du et al [38], is a prospective Chinese study from the China Kadoorie Biobank of 512,000 subjects mean age 51 years. At baseline a computer-based method was used to collect information about frequency of consumption of red meat, poultry, fish, fresh fruit and other items. Also, at baseline, the participants reported regular consumption ([?] 4 days/week) of red meat by $47.0 \%$, poultry by $1.3 \%$, or fish by $9.9 \%$. After a mean follow-up of 9.0 years, and adjusting for adiposity and other confounders, each 
$50 \mathrm{~g} /$ day increase in consumption of red meat or fish was associated with $11 \%$ and $6 \%$ increase in risk for T2DM, HR 1.11 (95\% CI 1.04-1.20) and HR 1.06 (95\% CI 1.00-1.13) for red meat and fish, respectively. In addition, the association was higher in men and women from urban areas, HR 1.42 (95\% CI 1.15-1.74) for red meat and HR 1.18 (95\% CI 1.03-1.36) for fish, respectively compared to those living in non-urban areas. No association was noted between poultry consumption and risk for T2DM, HR 0.96 (95\% CI 0.83-1.12). The study by Pan et al [39] is also, a prospective cohort study involving 26,357 men from the Health Professionals Follow-up Study (HPFS, 196-2006)), 48,709 women from the Nurses Health Study (NHS, 1986-2006), and 74,077 women from the (NHS II, 191-2007) study, which evaluated the effects of red meat consumption with the incidence of T2DM. After a mean follow-up of 17.1 years, the increase in red meat intake by 0.5 servings/day from baseline, was associated with a $48 \%$ increase in the incidence of T2DM, pHR 1.48 (95\% CI 1.37-1.59) after multiple adjustments, compared to a control group, who did not change the red meat consumption. In contrast, reduction of red meat consumption by 0.5 servings/day from baseline, decreased the incidence of T2DM by $14 \%$ in the next 4 years, HR $1.14 \%$ (95\% CI 7\%-20\%). Also, the study by Bondinelli et al [40], is a prospective case-control study of 340,234 adult subjects on the association of meat consumption and incidence of T2DM in the EPIC-InterAct Study. After 11.7 years of follow-up, 12,403 cases of incident T2DM were identified. In this large study, a dose-response association between red meat consumption and incidence of T2DM was found. In quintiles 1-5, the unprocessed red meat consumption was $18.6 \mathrm{~g} /$ day, $32,8 \mathrm{~g} /$ day, $45.6 \mathrm{~g} /$ day, $58.7 \mathrm{~g} /$ day and $81.5 \mathrm{~g} /$ day. For Q1 the HR for new onset T2DM was 1.00, and for quintiles 2-5 the HRs for new onset T2DM was 1.16 (95\% CI 1.07-1.27), 1.26 (95\% CI 1.36-1.38), 1.40 (95\% CI 1.27-1.53), and 1.57 (95\% CI 1.42-1.73), $\mathrm{p}<0.0001$ ) for each $50 \mathrm{~g} /$ day increments of unprocessed red meat. The processed meat consumption for quintiles 1-5, was $19.4 \mathrm{~g} /$ day, $28.4 \mathrm{~g} /$ day, 32.7 $\mathrm{g} /$ day, $39.0 \mathrm{~g} /$ day, and $56.2 \mathrm{~g} /$ day, and the HRs for new onset T2DM, were 1.00, 1.13 (95\% CI 1.04-1.22), 1.20 (95\% CI 1.10-1.30), 1.36 (95\% CI 1.25-1.48), and 1.61 (95\% CI 1.47-1.76), p < 0.0001 for linear trend. This study confirms a positive association between high consumption of unprocessed and processed red meat and incidence of T2DM. In a review and meta-analysis, Tian et al [41] analyzed the effects of consumption of red meat as well as other foods on the incidence of T2DM. This review included 483,174 subjects of whom, 52,637 developed T2DM. In these subjects, the pRR for new onset T2DM was 1.22 (95\% CI 1.09-1.36) for unprocessed red meat consumption, and 1.39 (95\% CI 1.29-1.49) for processed meat consumption. In contrast, th pRR for new onset T2DM with consumption of other foods was, 0.96 (95\% CI 0.88-1.06) for plant protein, 1.03 (95\% CI 0.89-1.17) for fish, 1.03 (95\% CI 0,64-1.67) for eggs, and 0.89 (95\% CI for total dairy foods. This review clearly demonstrates the benefits of healthy food consumption on the incidence of new onset T2DM.

\section{The Pathophysiology of Atherosclerotic Heart Disease.}

The association of increased intake of saturated fats with atherosclerosis has been long established from animal studies originally, by the work of Ignatowski [42] in 1908 and subsequently by the work of other investigators [43-45]. Atherosclerosis is the most frequent underlying cause of CAD, and CVD and is the major cause of CV mortality worldwide accounting for 17.3 million deaths/year, or $31.5 \%$ of the total death rate globally in 203 [46]. Saturated fat is rich in LDL-C, which has been considered the culprit for the pathogenesis of atherosclerosis [47], confirmed by genetic [48], epidemiologic [49], and clinical studies [50]. Therefore, the rationale for decreasing the intake of saturated fats is solid on scientific grounds. Several studies have demonstrated that reducing the saturated fat content of the diet and replacing it with polyunsaturated fats has resulted in a decreased incidence of CVD [51,52]. Also, populations with low intake of saturated fats, like those in the East Asian and Mediterranean countries, as well as the consumption of other diets low in saturated fats has been associated with a low incidence of ASCVD [53-55]. Since the effect of LDL-C on the risk of future ASCVD appears to be causal as well as cumulative over time, the earlier lowering of LDL-C may result, proportionally, in greater long-term reduction of ASCVD. It has been demonstrated that reduction of LDL-C by $1 \mathrm{mmol} / \mathrm{L}(38.7 \mathrm{mg} / \mathrm{dl})$ will reduce the relative risk of future ASCVD by about $10 \%$ the $1^{\text {st }}$ year, $16 \%$ the $2^{\text {nd }}$ year and $20 \%$ the $3^{\text {rd }}$ year [49]. Another factor regarding the atherogenic effects of LDL, is its consistency and particle size. LDL is a spherical particle 22-29 $\mathrm{nm}$ in diameter, with a core of esterified cholesterol and triglyceride, a lipid coat of non-esterified cholesterol and 
phospholipid, and a structural apoB protein, which is recognized by the LDL receptors that remove LDL from the plasma [56]. This structure of LDL consists of small dense sizes and of larger less dense sizes, and several studies have suggested that the small dens size of LDL is more atherogenic $[57,58]$, because it is more prone to oxidation and has greater affinity for the proteoglycans of the arterial wall facilitating its entrance into the arterial wall [59]. Also, small size LDL is present in high risk patients with increased triglyceride and low HDL levels, diabetes, insulin resistance, obesity, and the metabolic syndrome [60-62]. However, other observational studies have shown that large size LDL is also, atherogenic and predicts a higher incidence of CVD [63,64], although the preponderance of evidence supports the atherogenic properties of small dense LDL. Perhaps this controversy may not be important clinically, since both low size and large size LDLs respond equally well to treatment with statins [64].

\section{Discussion}

The studies presented, have shown a positive and linear relationship of unprocessed and especially, processed red meat consumption with the incidence of CVD, CHD, stroke, HF, T2DM, and all-cause mortality.

Cardiovascular disease: Several studies have demonstrated a positive association of increased consumption of unprocessed red meat and in particular processed meat with an increased incidence of CVD, CHD, and death $[6,20-29]$, which in some studies were dose-dependent $[6,22,23,24]$. The association of high red meat consumption with CV complications has been attributed to its high content of cholesterol and saturated fats. Saturated fats are high in LDL-C, which has been considered the culprit for the development of ASCVD, CAD and CVD by genetic, epidemiologic, and clinical observational studies [2-12]. The association with consumption of foods high in saturated fat with the incidence of ASCVD has been demonstrated experimentally, first by the studies of Ignatowski [42] in 1908 and subsequently, by other studies [43-45]. In addition, the coexistence of type 2 diabetes mellitus (T2DM) and increased BP caused by the high salt content of processed meat contribute to the increased incidence of CVD and CHD [65]. Processed meat contains also nitrites, which are converted in the bowel into nitrosamines, which have a toxic effect on the pancreatic $\beta$-cells, and in addition, it contains advanced glycation and lipooxydation end products, that have diabetogenic effects. It also, contains high amounts of salt, which could increase the BP [66-68]. Therefore, people should decrease as much as possible the consumption of unprocessed red meat and particularly the consumption of processed red meat, since several studies have shown a dose-response relationship between red meat consumption and the incidence of CVD.

Stroke: Besides its association with ASCVD, red meat consumption and especially processed meat has also, been associated with an increased risk of ischemic stroke [20,30-32]. The increased incidence of stroke with the consumption of processed red meat is a direct consequence of ASCVD, hypertension, and T2D, which have been shown to be increased with the high consumption of processed red meat which is high in saturated fats and salt [61-67].

Heart Failure: Red meat consumption, especially processed red meat, is also, associated with an increased risk of HF [32- 35]. The increased risk of HF could be due to a combination of ASCVD, CVD, CHD, T2DM, and hypertension. Other studies have also shown that the increased consumption of processed meat with its high content of salt, could add to the increased incidence of HF [34]. However, the exact association of red meat consumption with the incidence of HF is not clear at present and more studies are needed to clarify this association.

Type 2 Diabetes Mellitus: Several studies have reported an association between unprocessed and processed red meat and increased incidence of T2DM [36-40], with some reviews and meta-analyses reporting a high quality of evidence [74-76], with the exception of a major review and meta-analysis of cohort studies, which found a poor association between red meat consumption and the incidence of T2DM, probably due to the poor quality of included studies [77]. The new onset of T2DM appears to be multifactorial and among the various factors are, the saturated fatty acids, sodium, advanced glycation end products (AGEs), nitrates/nitrites, heme iron, branched chain aminoacids, (BCAAs), endocrine disruptor chemicals (EDCs), and the trimethylamine N-oxide (TMAO) derived from the conversion of choline in the gut [78-80]. 
Recent Trends in Food Selection: Red meat consumption is high the developed countries and is increasing in the developing countries. Of the 190 metric tons of meat produced globally each year, $\frac{1}{2}$ of it will be consumed by less than $25 \%$ of the people living in developed countries [75]. People living in Australia, UK and the US are among those consuming the highest quantities of red meat $[1,81]$. However, recent studies show that there is a healthy trend in decreasing red meat consumption by the developed countries, but a worrisome trend in increasing red meat consumption by the developing economies $[82,83]$. Indeed, in the US there is a recent tendency for plant-based meat consumption due to increased awareness of the adverse CV effects of animal meat [84]. An analysis of results of a prospective cohort study of 131,342 subjects of 46,329 were men from the US Health Care Professionals Study and 85,013 were women from the Nurses' Health Study, showed that plant-based meat consumption was associated with lower incidence of CV mortality than animal-based meat [85]. The public interest in plant-based protein has increased lately, and there is a resurgence in vegetarian and vegan diets [19] mostly by young people (38-65 years of age), usually female, educated ([?] high school education), physically active, and from the Western US regions [86]. Vegetarians usually follow a diet that does not include animal meat, fowl, seafood, or products containing these type of foods. Some vegetarians eat foods that contain dairy products (lacto-vegetarianism) or eggs (ovo-vetarianism), whereas the vegans eat foods that do not contain any type of animal meat, fish, dairy products, eggs, and sometimes even honey [86]. However, the percentage of vegetarians and vegans in the US was only $1.9 \%$ in 2012 and the great majority of people switching from red meat to white meat and plant-based meat were the previously red meat eaters. Based on this new trend, several companies have produced plant-based meat patties, which are sold by many fast food restaurants and supermarkets. Also, there are some studies demonstrating that animal meat from grassfed animals is leaner and healthier than meat from animals fed highly processed foods and it should be preferred for consumption [87]. The authors contend that this type of lean meat lowers the amount of saturated fats and is less prone to oxidation and peroxydation, which are implicated in the cause of CVDs and cancer [87]. Other studies contend that switching to consumption of plant-based meat and fiber-based products, although healthier, it could have significant economical consequences for the US, since the livestock industry employs $1.6 \times 10^{6}$ people and accounts for $\$ 31.8$ billion $\mathrm{kg}$ in meat exports, and that the livestock recycle more than $43.2 \times 10^{3} \mathrm{~kg}$ of human inedible food converting it into human edible food, pet food, industrial products, and $4 \times 10^{9} \mathrm{~kg}$ of nitrogen fertilizer [88]. Another contention of this study is that plant-based foods do not provide the necessary nutrients and energy required for the normal functions of the body. However, this contention has been challenged by studies showing that the quality of life of female and male vegetarian and vegan endurance runners is not different compared to omnivore runners [20]. We believe that the adaption of the recommendations by American College of Cardiology/American Heart Association (ACC/AHA) [89], to eat plant-based diets high in fiber, eggs, dairy products and unsaturated fatty acids, will far exceed the income reduction from the decreased consumption of animal-based meat. The conviction that high consumption of red meat is unhealthy is so strong, that a recent article by the Annals of Internal Medicine [90], suggesting that red meat consumption is not associated with adverse CV effects, was severely challenged by several investigators for reporting unsubstantiated and misleading information to the point of exerting legal action against the journal [91]. Of interest, are two recent studies [92,93] suggesting that the food industry by the year 2050 will have to feed 10 billion subjects and this will necessitate certain dietary changes in order to be sustainable. To accomplish this, a drastic change in the population diet will have to be made that will include the use of much more plant-based diet, and little red meat. Alternatively, they propose the adoption of a holistic flexitarian diet, which is a mixture of plant-based and animal-based foods and has been shown to have significant health benefits [94]..

Conclusion: The analysis of results from the selected papers presented, demonstrates that the consumption of red meat, processed and unprocessed, but not the white meat are associated with increased incidence of ASCVD, CVD, CHD, Stroke, HF and T2DM. Red meat consumption Is still high in the developed countries, although there is a tendency towards a decreased consumption lately. In contrast there is a progressive increase in its consumption by the developing countries, and this is a worrisome sign. On the other hand, the consumption .of healthier foods, like fiber-based products, dairy products, eggs, and unsaturated fats, is still limited. However, there is a recent tendency for an increased consumption of these products including the consumption of plant-based protein. The ACC/AHA guidelines, hopefully, will enforce this tendency 
and result in the decreased incidence of CVD, HF, stroke, and T2DM.

\section{References}

1. Daniel CR, Cross AJ, Koebnick c, et al. Trends in meat consumption in the USA. Public Health Nutr 2011; 14: 575- 583

2. Walker P, Rhubart-Berg P, McKenzie S, et al. Public health implications of meat production and consumption. Public Health Nutr 2005; 8: 348- 356

3. Speedy AW. Global production and consumption of animal source foods. J Nutr 2003; 133: 4048S4053

4. Rohrmann S, Overvad K, Bueno-Mesquita HB, et al. Meat consumption and mortality-results from the European Prospective Investigation into cancer and nutrition. BMC Med 2013; 1163

5. Sinha R, Cross AJ, Graubard BI, et al. Meat intake and mortality: a prospective study of over half a million people. Arh Intern Med 2009; 169: 562- 571

6. Zheng Y, Li Y, Satija A, et al. Association of changes in red meat consumption with total and cause specific mortality among US women and men: two prospective cohort studies. BMJ 2019; 365: I2110

7. Amiano P, Chamosa S, Etxezarreta N, et al. Unprocessed red meat and processed meat consumption and risk of stroke in the Spanish cohort of the European Prospective Investigation into Cancer and Nutrition (EPIC). Eur J Clin Nutr 2016; 70: 313- 319

8. Duncan MS, Vasan RV, Xanthakis V. Trajectories of blood lipid concentrations over the adult life course and risk of cardiovascular disease and all-cause mortality: observations from the Framingham study over 35 years. J Am Heart Assoc 2019; 8: e011433

9. Ference BA, Ginsberg HN, Graham I, et al. Low-density lipoproteins cause atherosclerotic cardiovascular disease. 1. Evidence from genetic, epidemiologic, and clinical studies. A consensus statement from the European Atherosclerosis Society Consensus Panel. Eur Heart J 2017; 38: 2459- 2472

10. Kim Y, Keogh J, Clifton P. A review of potential metabolic etiologies of the observed association between red meat consumption and development of type 2 diabetes mellitus. Metabolism 2015; 64: 768-779

11. Park K, Son J, Kang R, et al. Unprocessed meat consumption and incident cardiovascular diseases in Korean adults: the Korean Genome and Epidemiology Study (KoGES). Nutrients 2017; 9: 498

12. Yip CSC, Fielding R. A summary of meat intakes and health burdens. Eur J Clin Nutr 2018; 72: 1829

13. Stone NJ, Robinson JG, Lichtenstein AH, et al. Treatment of blood cholesterol to reduce atherosclerotic cardiovascular disease risk in adults. Synopsis of the 2013 American College of Cardiology/American Heart Association cholesterol guideline. Arh Intern Med 2014; 160: 339- 343

14. Mihaylova B, Enberson J, Blackwell L, et al. The effects of lowering LDL cholesterol with statin therapy in people at low risk of vascular disease: meta-analysis of individual data from 27 randomised trials. Cholesterol Treatment Trialists (CTT) Collaborators. Lancet 2011; 380: 581- 590

15. Ray KK, Seshasai SR, Ergou S, et al. Statins and all-cause mortality in high risk primary prevention: a meta-analysis of 11 randomized controlled trials involving 65,229 participants. Arch Intern Med 2010; 170: 1024- 1031

16. Yusuf S, Bosch J, Dagenais G, et al. Cholesterol lowering in intermediate-risk persons without cardiovascular disease. N Engl J Med 2016; 374: 2021- 2031

17. Grundy SM, Stone NJ, Bailey AL et al. 2018 AHA/ACC guideline on the management of blood cholesterol. A report from the American College of Cardiology/American Heart Association Task Force on Clinical Practice Guidelines. Circulation 2018;

18. Cramer H, Kessler CS, Sundberg T, et al. Characteristics of Americans choosing vegetarian and vegan diets for health reasons. J Nutr Educ Behav 2017; 49: 561- 567

19. Karlsen MC, Rogers G, Miki A, et al. Theoretical food and nutrient composition of whole-food plantbased and vegan diets compared to current dietary recommendations. Nutrients 2019; 11: 625

20. Boldt P, Knechtle B, Nikolaidis P, et al. Quality of life of female and male vegetarian and vegan endurance runners compared to omnivores-results from the NURMI study (steo 2). J Int Sports Nutr 
2018; 15: 33

21. Pan A, Bernstein AM, Schultze MB, et al. Red meat consumption and mortality. Results from 2 prospective cohort studies. Ann Intern Med 2012; 172: 555-563

22. Wang D, Campos H, Baylin A. Red meat intake is positively associated with non-fatal acute myocardial infarction in the Costa RicaHeart Study. Brit J Nutr 2017; 118: 303- 311

23. Key TJ, Appleby PN, Breadbury KE, et al. Consumption of meat, fish, dairy products, and eggs and risk of ischemic heart disease. A prospective study of 7198 incident cases among 409,885 participants in the Pan-European EPIC Cohort. Circulation 2019; 139: 2835- 2845

24. Alshahrami SM, Fraser GE, Sabate J, et al. Red and processed meat and mortality in low meaty intake population. Nutrients 2019; 11: 622

25. Zhong VW, Van Horn L, Greenland P, et al. Associations of processed meat, unprocessed red meat, poultry, or fish intake with incident cardiovascular disease and all-cause mortality. JAMA Intern Med 2020; 180: 503- 512

26. Micha R, Wallace SK, Mozaffarian D. Red and processed meat consumption and risk of incident coronary heart disease, stroke, and diabetes mellitus: a systematic review and meta-analysis. Circulation 2010; 121: 2271- 2283

27. Larsson SC, Orsini N. Red meat and processed meat consumption and all-cause mortality. Am J Epidemiol 2014;179: 282- 289

28. Bellavia A, Larsson SC, Bottai M, et al. Differences in survival associated with processed and with unprocessed red meat consumption. Am J Clin Nutr 2014; 100: 24- 29

29. Abete I, Bomaguera D, Vieira AR, et al. Association between total, processed, red and white meat consumption and all-cause, CVD and IHD mortality: a meta-analysis of cohort studies. Brit J Nutr 2014; 112: 762-

30. Chen GC, Pang Z, Liu QF. Red and processed meat consumption and risk of stroke: a meta-analysis of prospective cohort studies. Eur J Clin Nutr 2013; 67: 91- 95

31. Yang C, Pan L, Sun C, et al. Red meat consumption and the risk of stroke: A dose-response metaanalysis of prospective cohort studies. J Stroke Cerebrovasc Dis 2016; 25: 1177- 1186

32. Kim K, Hyeon J, Ah S, et al. Role of total, processed, and white meat consumption in stroke incidence and mortality: a systematic review and meta-analysis of prospective cohort studies. J Am Heart Assoc 2017; 6: e005983

33. Ashaye A, Gaziano J, Djousse L. Red meat consumption and risk of heart failure in male physicians. Nutr Metabol Cardiovasc Dis 2011; 21: 941- 946

34. Kaluza J, Akesson A, Wolk A. Processed and unprocessed red meat consumption and risk of heart failure. A prospective study of men. Circ Heart Fail 2014; 7: 552- 557

35. Kaluza J, Akesson A, Wolk A, Long-term processed and unprocessed red meat consumption and risk of heart failure: a prospective cohort study of women, Int J Cardiol 2015; 193: 42- 46

36. Cui K, Liu Y, Zhu L, et al. Association between intake of red and processed meat and the risk of heart failure: a meta-analysis. BMC Public Health 2019; 19: 354

37. Liu G, Zong G, Hu FB, et al. Cooking Methods for red meats and risk of type 2 diabetes: A prospective study of US women. Diabetes Care 2017; 40: 1041- 1049

38. Du H, Gao Y, Bennett DA, et al. Red meat, poultry and fish consumption and risk of diabetes: a 9 year prospective study cohort study of China Kadoorie Biobank. Diabetologia 2020; doi 10,1007

39. Pan A, Sun Q, Bernstein AM, et al. Changes in red meat consumption and subsequent risk of type 2 diabetes: three cohorts of US men and women. JAMA Intern Med 2013; 173: 1328- 1335

40. Bondinelli B, Palli D, Masala G, et al. Association between dietary meat consumption and incident type 2 diabetes: the EPIC-InterAct study. Diabetologia 2013; 56: 47- 59

41. Tian $\mathrm{S}, \mathrm{Xu} \mathrm{Q}$, Jiang R, et al. Dietary protein consumption and the risk of typ 2 diabetes: a systematic review and meta-analysis of cohort studies. Nutrients 2017; 9: 982

42. Konstantinov IE, Jankovic GM. Alexander I. Ignatowski. A pioneer in the study of atherosclerosis. Tex Heart Institute J 2013; 40: 246- 249

43. Armstrong ML, Heistad DD. Animal models of atherosclerosis. Atherosclerosis 1990; 85: 15- 23 
44. Li X, Liu Y, Zhang H, et al. Animal models of atherosclerosis research: a review. Protein Cell 2011; 2: $189-201$

45. Kapourchali FR, Surendiran G, Chen L, et al. Animal models of atherosclerosis. World J Clin Cases. 2014; 2: 126- 132

46. Sacks FM, Lichtenstein AH, Wu JKHY, et al. Dietary fats and cardiovascular disease. A Presidential Advisory from the American Heart Association. Circulation 2017; 136: e1-e23

47. Ference BA, Ginsberg HN, Graham I, et al. Low-density lipoproteins cause atherosclerotic cardiovascular disease. 1. Evidence from genetic, epidemiologic, and clinical studies. A consensus statement from the European Atherosclerosis Society Consensus Panel. Eur Heart J 2017;; 38: 2459- 2472

48. Nordestgaar BG, Chapman MJ, Humphries SE, et al. European Atherosclerosis Society Consensus Panel. Familial hypercholesterolemia is underdiagnosed and undertreated in the general population: guidance for clinicians to prevent coronary heart disease. Consensus Statement of the European Atherosclerosis Society. Eur Heart J 2013; 34: 3478- 3490

49. Di Angelantonio E, Gao P, Pennelis L, et al. Lipid-related markers and cardiovascular disease prediction. JAMA 2012; 307: 2499- 2506

50. Baigent C, Blackwell L, Emberson J, et al. Efficacy and safety of more than intensive lowering of LDL cholesterol : a meta-analysis of data from 170,000 participants in 26 randomized trials. Cholesterol Treatment Trialists (CTT). Lancet 2010; 376: 1670- 1681

51. Mozaffarian D, Micha R, Wallace S. Effects on coronary heart disease of increasing polyunsaturated fat in place of saturated fat: a systematic review and meta-analysis of randomized controlled trials. PloS Med 2010; 7: e1000252

52. Hooper L, Martin N, Abdelhamid A. Reduction in saturated fat intake for cardiovascular disease. Cochrane Database Syst Rev. 2015; CDO 11737

53. Farvid MS, Ding M, Pan A, et al. Dietary linoleic acid and risk of coronary heart disease; a systematic review and meta-analysis of prospective cohort studies. Circulation 2014; 130: 1568- 1578

54. Vardavas CI, Linardakis MK, Hatzis CM, et al. Cardiovascular disease risk factors and dietary habits of farmers from Crete 45 years after the first description of the Mediterranean diet. Eur J Cardiovasc Prev Rehabbil 2010; 17: 440- 446

55. Guash-Ferre M, Satija A, Blondin SA, et al. Meta-analysis of randomized controlled trials of red meat consumption in comparison with various comparison diets on cardiovascular risk factors. Circulation 2019; 139: 1828- 1845

56. Sacks FM, Campos H. Low-density lipoprotein size and cardiovascular disease: a reappraisal. J Clin Endocrinol Metabol 2003; 88: 4525- 4532

57. Lamarche B, Lemieux I, Despres JP. The small, dense LDL, phenotype and the risk of coronary heart disease : epidemiology, pathophysiology, and therapeutic aspects. Diabetes Metab 1999; 25: 199- 211

58. Austin MA. Triglyceride, small, dense low-density lipoprotein, and the atherogenic lipoprotein phenotype. Curr Atheroscler Rep 2000; 2: 200- 207

59. Skalen K, Gustafsson M, Knutsen Ryberg E, et al. Subendothelial retention of atherogenic lipoproteins in early atherosclerosis. Nature 2002; 417: 750- 753

60. McNamara JR, Campos H, Ordovas JM, et al. Effect of gender, age, and lipid status on low density lipoprotein subfraction distribution. Results of the Framingham Offspring Study. Arteriosclerosis 1987; 7: 483-

61. Austin MA, King MC, Vranizan KM, et al. Atherogenic lipoprotein phenotype: a proposed genetic marker for coronary heart disease risk. Circulation 1990; 82: 495- 506

62. Reaven GM, Chen YD, Jeppesen J, et al. Insulin resistance and hyperinsulinemia in individuals with small, dense low density lipoprotein particles. J Clin Invest 1993; 92: 141- 146

63. Campos H, Roederer GO, Lussier-Cacan S, et al. Predominace of large LDL and reduced HDL2 cholesterol in normolipidemic men with coronary artery disease. Arterioscl Thromb Vasc Biol 1995; 15: $1043-1048$

64. Campos H, Moye LA, Glasser SP, et al. Low-density lipoprotein size, pravastatin treatment, and coronary events. JAMA 2001; 286: 1468- 1474 
65. Haring B, Wang W, Fretts A, et al. Red meat consumption and cardiovascular target organ damage (from the Strong Heart Study). J Hypertens 2017; 35: 1794- 1800

66. Bronzato S, Durante A. Contemporary review of the relationship between red meat consumption and cardiovascular risk. Int J Prev Med 2017; 8: 40

67. Feskens EJM, Sluik D, van Woudenbergh. Meat consumption, diabetes, and its complications. Curr Diab Rep 2013; 13: 298- 306

68. Van Dam RM, Willett WC, Rimm EB, et al. Dietary fat and meat intake in relation to risk of type 2 diabetes in men. Diabetes Care 2002; 25: 417- 424

69. Viassara H, Bucalla R, Striker L. Pathogenic effects of advanced glycosylation: biological and clinical implications for diabetes and aging. Lab Invest 1994; 70: 138- 151

70. Colditz GA, Manson JE, Sampfer MJ, et al. Diet and risk of clinical diabetes in women. Am J Clin Nutr 1992; 55: 1018- 1023

71. Wu PY, Yang SH, Wong TC, et al. Association of processed meat intake with hypertension risk in hemodialysis patients: A cross-sectional study. PLoS One 2015; 10: e141917

72. Steffen LM, Kroenke CH, Yu X, et al. Association of plant food, dairy product, and meat intakes with 15-y incidence of elevated blood pressure in young black and white adults: the Coronary Artery Risk Development in Young Adults (CARDIA) Study. Am J Clin Nutr 2005; 82: 1169- 1177

73. Battaglia Richi E, Baumer B, Conrad B, et al. Health risks associated with meat consumption: a review of epidemiological studies. Int J Vitam Nutr Res 2015; 85: 70-78

74. Feskens EJM, van Woudenbergh GJ. Meat consumption, diabetes, and its complications. Curr Diab Rep 2013; 13: 298- 306

75. Schwingshacki L, Hoffmann G, Lampousi AM, et al. Food groups and risk of type 2 diabetes mellitus: a systematic review and meta-analysis of prospective studies. Eur J Epidemiol 2017; 32: 363- 375

76. Neuenshwander M, Ballon A, Weber KS, et al. Role of diet in type 2 diabetes incidence: umbrella review of meta-analyses of prospective observational studies. BMJ 2019; 365: 12368

77. Zeraatkar D, Han MA, Guyatt GH, et al. Red and processed meat consumption and risk for all-cause mortality and cardiometabolic outcomes. Ann Intern Med 2019; 171: 703- 710

78. Yang JJ, Lipworth LP, Shu XO, et al. Associations of choline-related nutrients with cardiometabolic and all-cause mortality: results from 3 prospective cohort studies of blacks, whites, and Chinese. Am J Clin Nutr 2020; 111: 644- 656

79. Roy S, Yuzefpolskaya M, Nandakumar R, et al. Plasma trimethylamine-N-oxide and impaired glucose regulation: results from the Oral Infections, Glucose Intolerance and Insulin Resistance Study (ORIGINS). PloS One 2020; 15: e0227482

80. Yoon NS. The emerging role of branched-chain amino acids in insulin resistance and metabolism. Nutrients 2016; 8: 405

81. O'Leary F, Grech A, Sui Z, et al. Older Australians are eating more protein: secondary analysis of the 1995 \& 2011 national nutrition surveys. Eur J Clin Nutr 2019; August 5

82. Santeramo FG, Carlucci D, De Devitiis B, et al. Emerging trends in European food, diets and food industry. Food Res Int 2018; 104: 39- 47

83. Vranken L, Averaete T, Petalios D, et al. Curbing global meat consumption: emerging evidence of a second nutrition transition. Enviromental Sci Policy 2014; 39: 95- 106

84. Mehta N, Ahlawat SS, Dabur RS. Novel trends in development of dietary fiber rich meat products-a critical review. J Food Sci Technol 2015; 52: 633- 647

85. Song M, Fung TT, Hu FB, et al. Association of animal and plant protein intake with all-cause and cause specific mortality. JAMA Intern Med 2016; 176: 1453- 1463

86. Cramer H, Kessler CS, Sundberg T, et al. Characteristics of Americans choosing vegetarian and vegan diets for health reasons. J Nutr Educ Behav 2017; 49: 561- 567

87. Provenza FD, Kronberg SL, Gregorini P. Is grassfed meat and dairy better for human and environmental health?. Front Nutr 2019; 6: 26

88. White RR, Hall MB. Nutritional and greenhouse gas impacts of removing animals from US agriculture. Proc Natl Acad Sci, USA 2017; 114: E10301- E10308 
89. Stone NJ, Robinson JG, Lichtenstein AH, et al. Treatment of blood cholesterol to reduce atherosclerotic cardiovascular disease risk in adults: synopsis of the 2013 American College of Cardiology/American Heart Association cholesterol guideline. Ann Intern Med 2014; 160: 339- 343

90. Johnston BC, Zeraatkar D, Han MA, et al. Unprocessed red meat and processed meat consumption: Dietary Guideline Recommendations from the Nutritional Recommendations (NutriRECS) Consortium. Ann Intern Med 2019; doi:10: 7226/M 19-1621

91. Rubin R. Backlash over meat dietary recommendations raises questions about corporate ties to nutrition scientists. JAMA 2020; doi: 1001/JAMA 2019: 21441

92. Schmidt CV, Mouritsen OG. The solution to msustainable eating is not a one-way street. Front Psychol 2020; 11: 531

93. Willett W, Rockstrom J, Loken B, et al. Food on the anthropocene: the EAT-Lancet commission on healthy diets from sustainable food systems. Lancet 393: 447- 492

94. Derbyshire EJ. Flexitatian diets and health: a review of the evidence-based literature. Front Nutr 2017; 3: 56

\section{Hosted file}

Table 1. Red meat intake and cardiovascular disease.docx available at https://authorea.com/ users/332322/articles/458781-association-of-red-meat-consumption-with-the-incidence-ofcardiovascular-disease

\section{Hosted file}

Table 2. Red meat consumption and Incidence of Stroke.docx available at https://authorea. com/users/332322/articles/458781-association-of-red-meat-consumption-with-the-incidenceof-cardiovascular-disease

\section{Hosted file}

Table 3.Red meat red consumption and Risk of Heart Failure.docx available at https: //authorea.com/users/332322/articles/458781-association-of-red-meat-consumption-withthe-incidence-of-cardiovascular-disease

\section{Hosted file}

Table 4. Red Meat Consumption and T2DM.docx available at https://authorea.com/users/ 332322/articles/458781-association-of-red-meat-consumption-with-the-incidence-ofcardiovascular-disease 